\title{
The long non-coding RNA ENST00000547547 reduces 5-fluorouracil resistance of colorectal cancer cells via competitive binding to microRNA-31
}

\author{
JIAN LI ${ }^{1,2}$, XIAORONG LI ${ }^{1}$, CHAOQUN CEN ${ }^{3}$, XIAOHUI AI ${ }^{5}$, CHANGWEI LIN ${ }^{1}$ and GUI HU ${ }^{4}$ \\ Departments of ${ }^{1}$ Gastrointestinal and Thyroid Surgery, ${ }^{2}$ Nuclear Medicine, ${ }^{3}$ Emergency Medicine and ${ }^{4}$ General Surgery, \\ The Third Xiangya Hospital of Central South University, Changsha, Hunan 410013; \\ ${ }^{5}$ Department of Oncological Surgery, Traditional Chinese Medicine of \\ Shaoyang Hospital, Shaoyang, Hunan 422001, P.R. China
}

Received April 21, 2017; Accepted October 13, 2017

DOI: 10.3892/or.2017.6082

\begin{abstract}
Colorectal cancer (CRC) is one of the most common cancers and the third leading cause of cancer-related deaths due to its rapid progression and poor prognosis. 5-Fluorouracil (5-FU)-based chemotherapies are the standard treatment for locally advanced CRC. However, a considerable percentage of CRCs have inherent or acquired 5-FU resistance, which critically impedes clinical outcomes. In the present study, we reported that the expression level ENST00000547547 was downregulated in 5-FU-resistant CRC cells in comparison with the parental cells, While rising with the treatment of 5-FU in parental cells. Overexpression of ENST00000547547 promoted 5-FU-induced cell apoptosis and reduced the chemoresistance of 5-FU in vitro. Moreover, we found that ENST00000547547 was a target of miR-31, as confirmed by dual-luciferase reporter assay and RNA immunoprecipitation assay. Notably, miR-31 was upregulated in 5-FU-resistant CRC cells, and knockdown of miR-31 increased the chemosensitivity of 5-FU-resistant CRC cells. Furthermore, we demonstrated that ENST00000547547 reduced the chemoresistance of 5-FU via competitive binding to miR-31 in 5-FU-resistant CRC cell lines. Collectively, our findings revealed that ENST00000547547 reduced chemoresistance in 5-FU of 5-FU-resistant CRC cells through competitive binding to miR-31 and has the potential to serve as a therapeutic target in CRC patients.
\end{abstract}

\section{Introduction}

Colorectal cancer (CRC) is the third most common cancer and one of the leading causes of cancer-related deaths worldwide.

Correspondence to: Dr Gui Hu, Department of General Surgery, The Third Xiangya Hospital of Central South University, Tongzipo Road, Changsha, Hunan 410013, P.R. China

E-mail: guihu_csu@126.com

Key words: long non-coding RNA, ENST00000547547, microRNA, miR-31, 5-FU-based drug resistance
According to WHO GLOBOCAN 2012, 1.4 million new cases of CRC were diagnosed, and 0.7 million patients succumbed to CRC, which accounted for $9.7 \%$ of the total cancer incidence and $8.5 \%$ of the cancer-related deaths worldwide (1). 5-Fluorouracil (5-FU), firstly synthesized by Heidelberger et al in 1957, has been widely employed for the treatment of solid cancers $(2,3)$. The 5-FU-based chemotherapy is the standard treatment for locally advanced CRC, particularly for middle and distal rectal cancers, which improves the response rate, local control and overall survival $(4,5)$. However, a considerable percentage of CRCs have inherent or acquired resistance to 5-FU-based chemotherapy, which is a responsible for the failure of the treatment $(6,7)$. Thus, a deeper understanding of the molecular characteristics underlying 5-FU resistance is imperative.

In recent years, numerous studies have indicated that long non-coding RNAs (lncRNAs), generally longer than 200 nucleotides in length, have emerged as new effective regulators in tumor initiation and progression (8-10). IncRNAs generally act as key molecules in chromatin remodeling, transcription, and post-transcriptional processing (11).The roles that IncRNAs play in drug resistance have also been extensively reported (12-15). Lan et al demonstrated that silencing of IncRNA ANRIL inhibited the development of multipledrug resistance in gastric cancer cells (16). Liu et al reported that overexpression of IncRNA PVT1 in ovarian cancer cells promoted cisplatin resistance through the regulation of apoptotic pathways (17). Wang et al indicated that increased expression of lncRNA UCA1 improved cell migration and invasion, and induced cisplatin resistance in human ovarian cancer cells (18). In CRC, several lncRNAs were found to be effective regulators of tumorigenesis, such as PRNCR1, MALAT-1, HOTAIR and AK027294, which partly or totally affected cell proliferation, metastasis, cell cycle progression, apoptosis and epithelial-mesenchymal transition (19-22). In 2015, a microarray analysis identified 2,662 differentially expressed lncRNAs in 5-FU-resistant CRC cells when compared with those in parental cells, suggesting that lncRNAs may play significant roles in the 5-FU resistance of CRC cells (23). However, a relative functional study has rarely been reported. 
lncRNA ENST00000547547 is a 434-bp transcript on human chromosome 12q15 (RP11-611E13.3-001) and it has been found to be significantly downregulated in drug-resistant CRC cells. A bioinformatic analysis indicated that ENST00000547547 contains a potential binding site for microRNA-31 (miR-31). miR-31 is a broadly conserved miRNA expressed in a variety of tissues and types of cells, that has been revealed to be upregulated in various human cancers, including breast, tongue and head-and-neck squamous tumors (24). Wang et al demonstrated that suppression of miR-31 in HCT116 cells increased its sensitivity to 5-FU at an early stage, and the downregulation of miR-31 affected cell migration and invasion (25). However, the potential association between ENST00000547547 and miR-31, and its functional role in 5-FU resistance of CRC remain largely unknown.

In the present study, we used 5-FU-resistant CRC cell models to determine the potential roles of ENST00000547547 and miR-31 in the regulation of 5-FU resistance. Following a functional study, we applied a dual-luciferase reporter assay and an RNA immunoprecipitation assay to examine the relationship between ENST00000547547 and miR-31. Finally, the functional role of the their interaction in 5-FU resistance was investigated using co-transfection cell models. Our results strongly indicated that ENST00000547547 reduces 5-FU resistance via competitive binding to miR-31 in 5-FU-resistant CRC cell lines and may provide new therapeutic targets for patients that exhibited 5-FU resistance.

\section{Materials and methods}

Cell culture and reagents. Human CRC cell lines HCT116 and LoVo, and 5-FU-resistant CRC cell lines LoVo/5-FU and HCT116/5-FU were purchased from Auragene (Changsha, China). The cells were cultured in Dulbecco's modified Eagle's medium (DMEM; HyClone, Logan, UT, USA) supplemented with $10 \%$ fetal bovine serum (FBS; Thermo Fisher Scientific, Waltham, MA, USA), $100 \mathrm{U} / \mathrm{ml}$ penicillin, and $100 \mathrm{mg} / \mathrm{ml}$ streptomycin (Invitrogen Life Technologies, Carlsbad, CA, USA). The cultures were maintained at $37^{\circ} \mathrm{C}$ in humidified air with $5 \% \mathrm{CO}_{2}$. 5-FU (F6627) was purchased from SigmaAldrich (St. Louis, MO, USA).

RNA extraction and $q R T-P C R$ analyses. Total RNA was extracted from frozen cells with TRIzol reagent (Invitrogen Life Technologies), and cDNA was synthesized using random primers and a Reverse Transcription kit (Takara Biotechnology, Dalian, China). The amount and quality of RNA were determined based on absorbance ratios using NanoDrop ND-1000. Quantitative RT-PCR (qRT-PCR) analyses were performed using SYBR-Green qPCR Mix (Toyobo, Osaka Prefecture, Osaka, Japan) according to the manufacturer's instructions. The expression levels of target genes were normalized to the transcription level of $\beta$-actin, and U6 was used for normalization of miR-31 expression. The data were collected and calculated using an ABI 7300 instrument (MK3; Thermo Fisher Scientific). Primers for qRT-PCR were synthesized by Invitrogen (Carlsbad, CA, USA) and the sequences were: ENST00000547547 sense, 5'-TTTTCTAAGGCACCA ACT-3' and antisense, 5'-CCAAA TGCTCTAAGGGA-3'; miR-31 primer was obtained from
FulenGen company (HmiRQP0401); ABCB9 sense, 5'-GCTCTGG GAGAGACCTTCCT-3' and antisense, 5'-GAGCGGAAGAGA CAGTTTCG-3'; $\beta$-actin sense, 5'-AGGGGCCGGACTCGTC ATACT-3' and antisense, 5'-GGCGGCACCACCATGTAC CCT-3'.

Transfection. Cells were transfected using transfection reagent Lipofectamine 2000 (Invitrogen Life Technologies) according to the manufacturer's instructions. They were harvested at $48 \mathrm{~h}$ after transfection. For co-transfection (pre-miR-31 and pCDNA3.1-ENST00000547547 as an example) the cells were initially transfected with $\mathrm{NC}$ or pre-miR-31 using Lipofectamine 2000, according to the protocols. Then, the cells were transfected with pCDNA3.1-ENST00000547547 or the mutant one using Lipofectamine 2000, according to the manufacturer's instructions.

Cell proliferation assay. The cell proliferation assay was conducted using 3-(4,5-dimethylthiazol-2-yl)-2,5-diphenyltetrazolium bromide (MTT) solution (Sangon Biotech, Shanghai, China). Forty-eight hours after transfection, these cells and controls were seeded into 96-well plates at an initial density of 5,000 cells/well. After $24 \mathrm{~h}$ of culture, these cells were exposed to various concentrations of 5-FU $(0,5,10,20,40,60,80$ and $100 \mu \mathrm{M}$ ) for $48 \mathrm{~h}$, and then they were treated with $10 \mu \mathrm{l}$ MTT by adding it into each well. The cells were incubated at $37^{\circ} \mathrm{C}$ for another $4 \mathrm{~h}$, then the medium was removed carefully, and $150 \mu 1$ dimethyl sulfoxide (DMSO) solution (MP Biomedicals, LLC, Santa Ana, CA, USA) was added to lyse the cells. Finally, the absorbance was assessed at $570 \mathrm{~nm}$ with a microplate reader Multiskan MK (Thermo Fisher Scientific). The survival rate was calculated using the equation: (mean absorbance of the drug wells/mean absorbance of the control wells) x $100 \%$.

Flow cytometric analysis. Cells were harvested $48 \mathrm{~h}$ after 5-FU treatments $(20 \mu \mathrm{M})$, and were washed three times with cold phosphate-buffered saline (PBS). Then, these cells were stained with $5 \mu \mathrm{l}$ Annexin V-FITC and $5 \mu \mathrm{l}$ propidium iodide (PI; Keygentec, Nanjing, China) for $10 \mathrm{~min}$ in the dark at room temperature. The cells were then examined by flow cytometry (BD Biosciences, Frankin Lakes, NJ, USA).

Western blot analysis. Proteins were extracted from cells with RIPA lysis buffer (Auragene, Changsha, China), which was supplemented with a protease inhibitor cocktail (Roche, Basel, Switzerland) and phenylmethylsulfonyl fluoride (PMSF) (Auragene, Changsha, China). Equal amounts $(30 \mu \mathrm{g})$ of proteins were loaded on sodium dodecyl sulfatepolyacrylamide gel electrophoresis (SDS-PAGE), and then were transferred to a polyvinylidene difluoride (PVDF) membrane (Millipore, Bedford, MA, USA). The membrane was blocked with $3 \%$ bovine serum albumin (BSA)-Trisbuffered saline with Tween-20 (TBST) with gentle shaking at room temperature for $90 \mathrm{~min}$ and was continuously probed with indicated primary antibodies with gentle shaking at $4^{\circ} \mathrm{C}$ overnight. The membranes were washed, and incubated with specific secondary antibodies at room temperature for $1 \mathrm{~h}$. A $\beta$-actin antibody was used as a control, and the ABCB9 (1:1,000; Santa Cruz Biotechnology, Inc., Dallas, TX, USA), Bcl-2 (1:1,000), Bax (1:500) (both from Abzoom, Dallas, TX, 

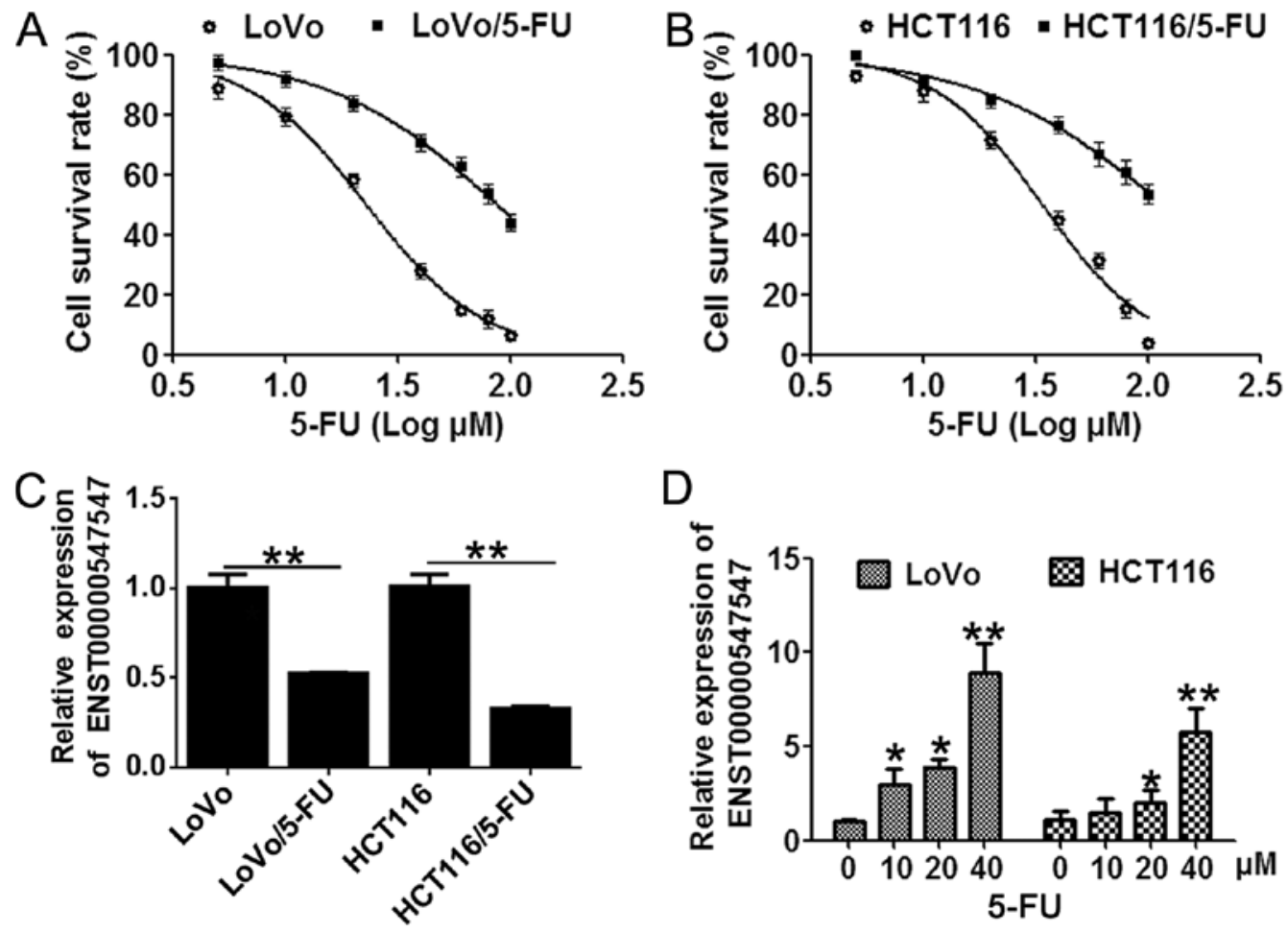

Figure 1. ENST00000547547 is downregulated in 5-FU-resistant CRC cell lines. (A and B) Parent and 5-FU-resistant CRC cells were exposed to various concentrations of 5-FU for $48 \mathrm{~h}$, and the cell survival rate was determined by MTT assay. (C) The transcriptional levels of ENST00000547547 in parent and 5-FU-resistant CRC cells were determined by qRT-PCR analysis. (D) Each cell line was exposed to various concentrations of 5-FU for $48 \mathrm{~h}$. Transcriptional levels of ENST00000547547 in CRC cell lines were determined by qRT-PCR analysis. Data represent the mean \pm SD from three independent experiments; ${ }^{*} \mathrm{P}<0.05,{ }^{* *} \mathrm{P}<0.01$.

USA), AGO2 (1:500) and IgG $(1: 1,000)$ (both from Abcam, Cambridge, MA, USA) antibodies were used for each group.

Bioinformatics analysis. miR-31 was predicted as a target of ENST00000547547 using bioinformation tool, miRcode. In addition, $\mathrm{ABCB} 9$ was reported to be targeted by miR-31 (microRNA.org) (26).

Dual-luciferase reporter assay. The fragments from ENST00000547547 containing the predicted miR-31 binding site were synthesized and cloned into the luciferase construct psi-CHECK2. The resulted vector ENST000005475473'UTR-psi-CHECK2 was called the reporter vector ENST00000547547-3'UTR. The corresponding mutant was called the reporter vector Mut-ENST00000547547. The pre-miR-31 or negative control pre-Con was co-transfected with the reporter vectors using transfection reagent (Invitrogen Life Technologies). Forty-eight hours after transfection, firefly and Renilla luciferase activities in cell lysates were assessed using the Dual-Luciferase Reporter Assay kit (Promega, Madison, WI, USA).

RNA immunoprecipitation (RIP) assay. The RIP assay for ENST00000547547 and miR-31 was carried out with the EZ-Magna RIP kit (Millipore) using $5 \mathrm{mg}$ of goat anti-AGO2 antibody (Santa Cruz Biotechnology, Inc.), according to the manufacturer's instructions. The IgG-MS2-based RIP assay was carried out using the Anti-IgG Affinity Gel (Sigma, St.Louis, MO, USA). The co-precipitated RNAs were extracted with TRIzol reagent (Invitrogen) and detected by qRT-PCR.
Statistical analysis. All statistical analyses were performed using SPSS 20.0 (IBM SPSS, Armonk, NY, USA) and GraphPad Prism 5 (GraphPad Software, La Jolla, CA, USA). Data are expressed as the mean \pm standard deviation (SD) from at least three independent experiments. The paired t-test was used for statistical analyses between two groups, and one-way analysis of variance (ANOVA) was used for statistical analyses among three groups. $\mathrm{P}<0.05$ was considered to indicate a statistically significant result.

\section{Results}

ENST00000547547 is downregulated in 5-FU-resistant CRC cell lines. In the present study, to confirm the chemosensitivity of 5-FU in 5-FU-resistant CRC and parent cells, an MTT assay was used for evaluation. It revealed that the cell survival rates of 5-FU-resistant CRC cell lines LoVo/5-FU (Fig. 1A) and HCT116/5-FU (Fig. 1B) were significantly higher than the parental cell lines when exposed to $5-\mathrm{FU}$. The $\mathrm{IC}_{50}$ values of 5-FU in LoVo/5-FU and HCT116/5-FU were 87.01 and $117.7 \mu \mathrm{M}$. However, the $\mathrm{IC}_{50}$ values of 5-FU in LoVo and HCT116 cells were 22.81 and $33.79 \mu \mathrm{M}$, which indicated 5-FU-resistant CRC cell lines were successfully constructed.

Then, we investigated the expression of ENST00000547547 in parental and 5-FU-resistant CRC cell lines by qRT-PCR (Fig. 1C). The results revealed that the expression level of ENST00000547547 was significantly downregulated in 5-FU-resistant CRC cell lines LoVo/5-FU and HCT116/5-FU compared with parental cells. Moreover, as shown in Fig. 1D, the expression of ENST00000547547 was elevated correlated 

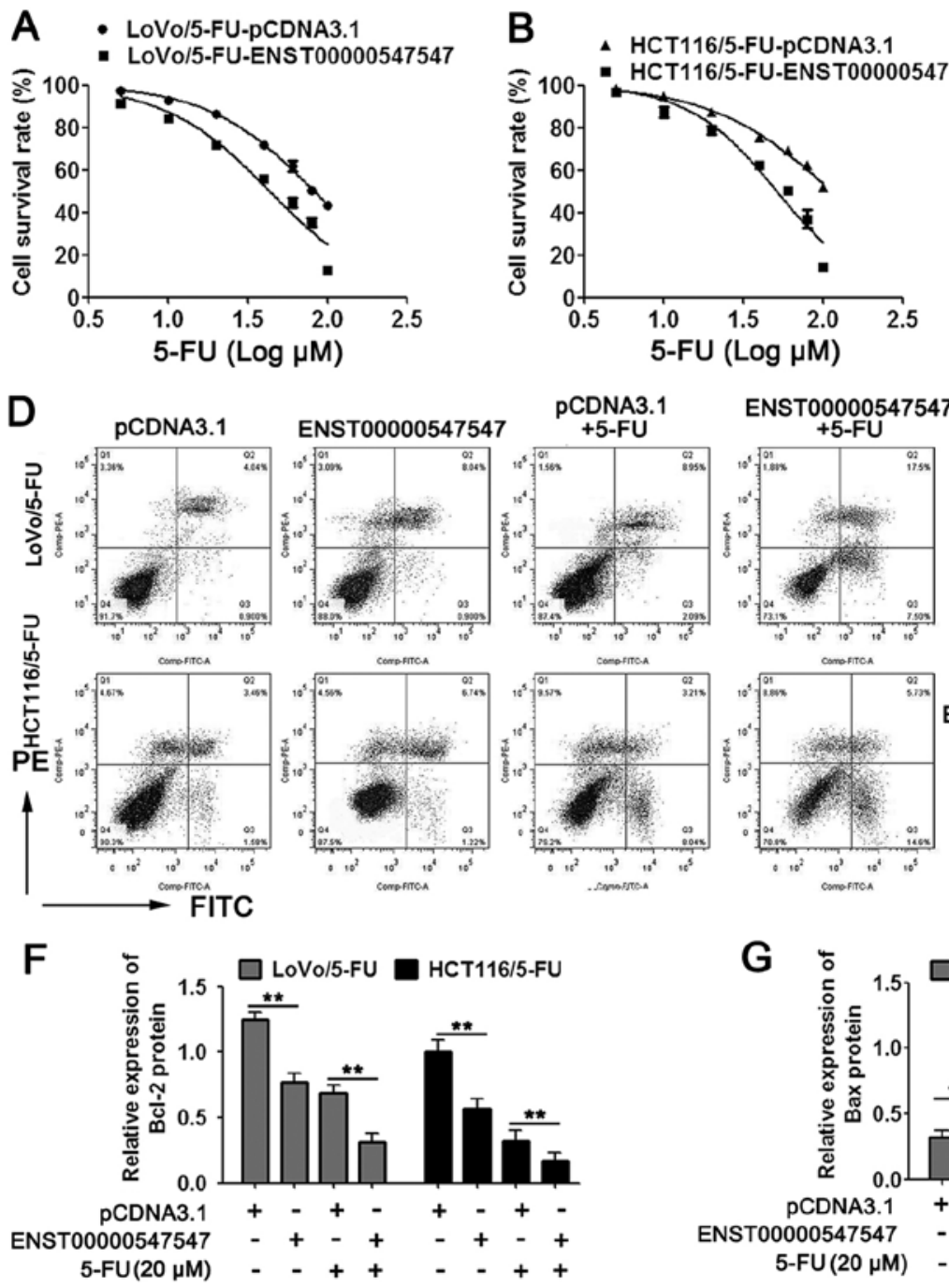

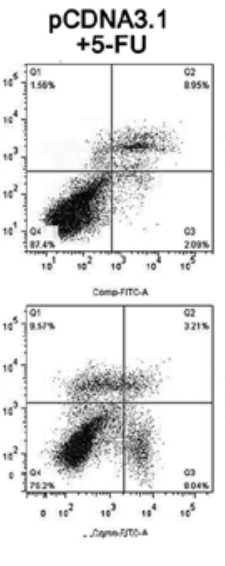

ENST00000547547

+5-FU
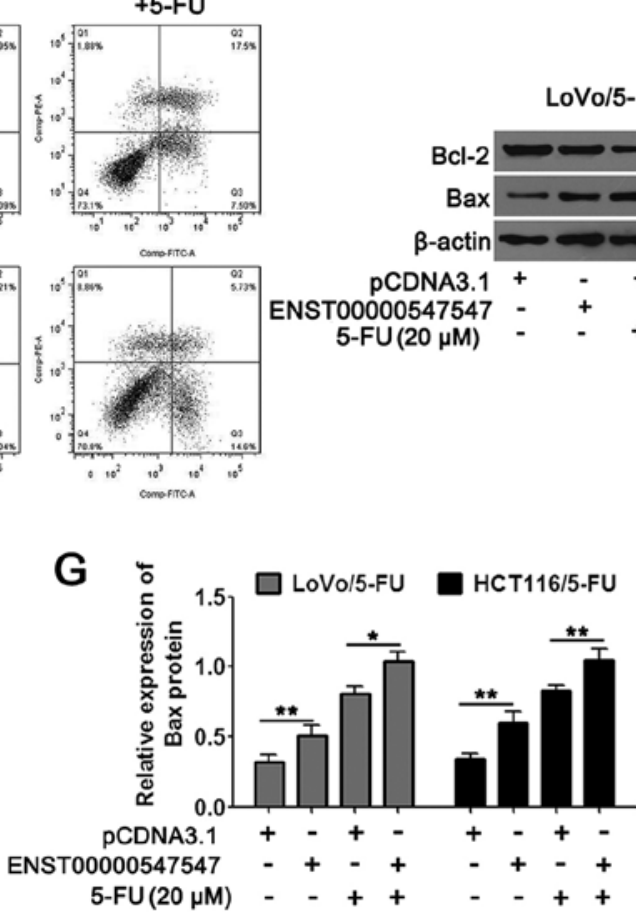

Figure 2. Overexpression of ENST00000547547 reduces the 5-FU resistance of CRC cell lines. (A and B) The cell survival rate was determined by MTT assay. All cell lines were exposed to various concentrations of 5-FU for $48 \mathrm{~h}$. (C and D) Flow cytometric assays were used to analyze the cell apoptosis in ENST00000547547 overexpressing CRC cell lines with or without 5-FU treatment. (E-G) The protein levels of BCL-2 and BAX were assessed by western blot analysis. All experiments were performed in biological triplicates; ${ }^{*} \mathrm{P}<0.05,{ }^{* *} \mathrm{P}<0.01$.

to the increasing dosage of 5-FU treatment in CRC cell lines. Collectively, these results indicated that ENST00000547547 was downregulated in 5-FU-resistant CRC cell lines and that it may play a role in 5-FU resistance.

Overexpression of ENST00000547547 reduces the 5-FU resistance of $C R C$ cell lines. To determine the potential biological function of ENST00000547547 in the 5-FU resistance of CRC cell lines, we applied overexpression vector pCDNA3.1-ENST00000547547 to upregulate the expressions of ENST00000547547 in HCT116/5-FU and LoVo/5-FU cells. The resistance of 5-FU was evaluated based on survival rate by MTT assay (Fig. 2A and B). The $\mathrm{IC}_{50}$ values of 5-FU in ENST00000547547-overexpressed LoVo/5-FU and HCT116/5-FU cells were $43.21 \mu \mathrm{M}$ and $51.31 \mu \mathrm{M}$ and the $\mathrm{IC}_{50}$ of 5-FU in LoVo/5-FU-pCDNA3.1 and HCT116/5-FU-pCDNA3.1 were $82.35 \mu \mathrm{M}$ and $114.2 \mu \mathrm{M}$. It was suggested that ENST00000547547 overexpression reduced 5-FU resistances in CRC cells. To further determine whether ENST00000547547 had an effect on 5-FU-induced cell apoptosis in 5-FU-resistant cells, flow cytometric assays were performed. The results revealed that overexpression of ENST00000547547 in HCT116/5-FU and LoVo/5-FUcells with 5-FU or without 5-FU treatment had higher apoptotic rates in comparison with the control cells (Fig. 2C and D). The Bcl-2 family members Bcl-2 and Bax are important anti- or pro-apoptotic regulators, respectively (27). To investigate whether the pro-apoptotic effect of ENST00000547547 was related to the Bcl-2 family, the expressions of Bcl-2 and Bax were examined. The western blot results revealed that Bcl-2 expression was decreased in ENST00000547547overexpressed cells, whereas the expression of Bax was increased compared with the control groups (Fig. 2E-G). Collectively, these findings indicated that overexpression of ENST00000547547 reduced the 5-FU resistance of CRC cell lines, and this decreased 5-FU resistance related to the proapoptotic effect of ENST00000547547 on CRC cells.

ENST00000547547 directly binds to miR-31 and suppresses its expression. To investigate the relationship between ENST00000547547 and miR-31, we performed dual-luciferase reporter and RIP assays. As shown in 
A

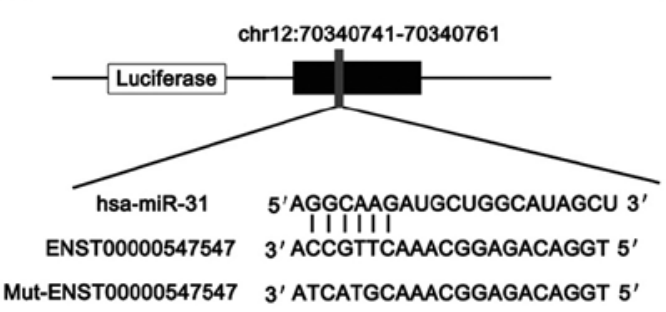

$\mathrm{B}$

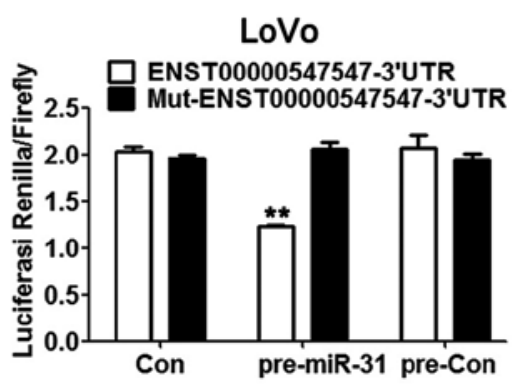

C

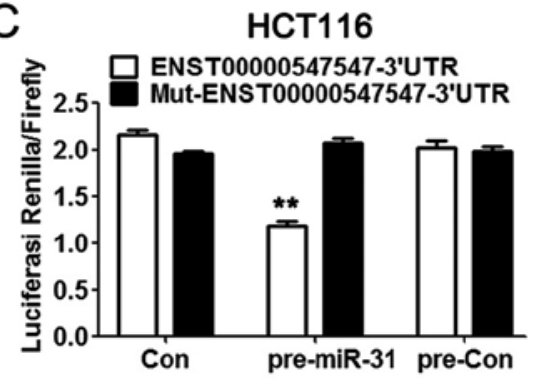

\section{D :}

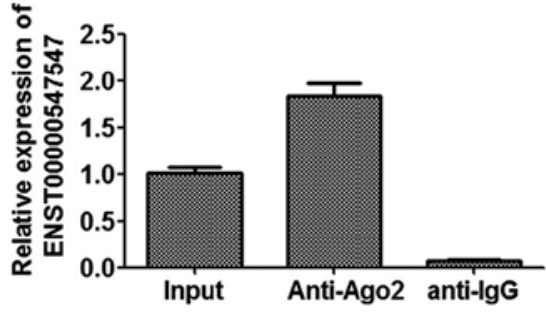

E :

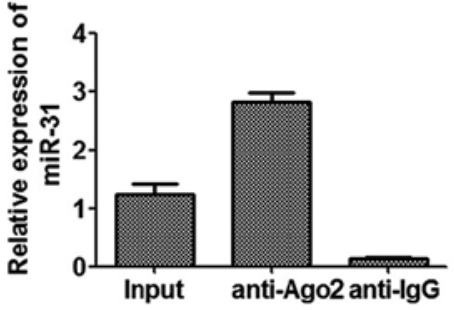

$\mathrm{F}$

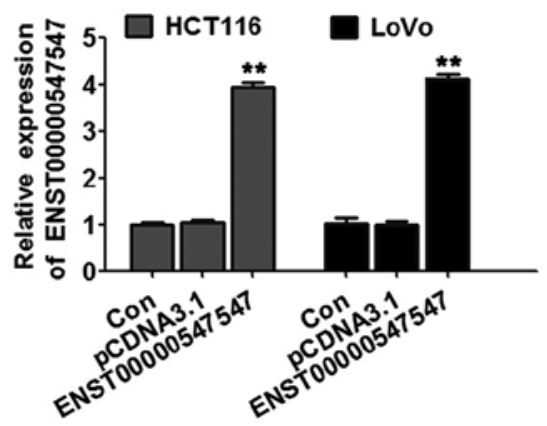

G

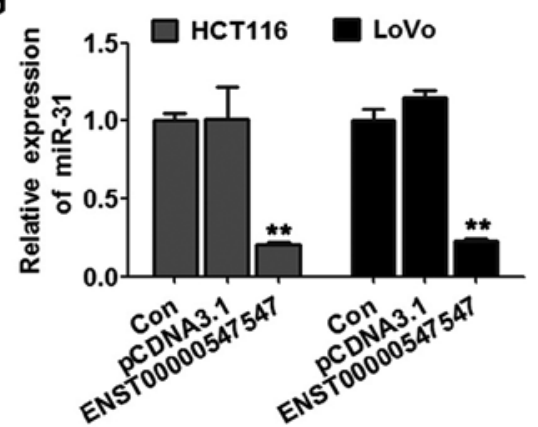

$\mathrm{H}$

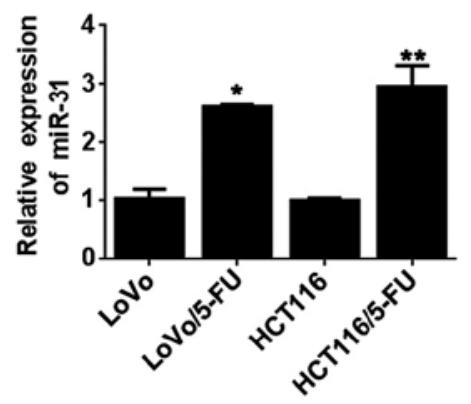

Figure 3. ENST00000547547 directly binds to miR-31 and suppresses its expression. (A) ENST00000547547 RNA contains a site complementary to miR31 , and the mutated form of ENST00000547547 sequence is revealed. (B and C) Luciferase activity was detected in CRC cells after transfection with miR-31 mimics (pre-miR-31) or miR-31 (negative control; pre-Con), and transfection with luciferase constructs of ENST00000547547-psi-CHECK2 or MutENST00000547547-psi-CHECK2. Cells without miRNA transfection were used as a blank control (Con). (D and E) RIP analysis was performed in (D) LoVo and (E) HCT116 cells after transfection with miR-31. IgG was used as a negative control. The ENST00000547547 mRNA level was detected in the substrate of the RIP assay by qRT-PCR. (F and G) The expression of ENST00000547547 and miR-31 in the Con, pCDNA3.1 and pCDNA3.1-ENST00000547547 groups was examined by qRT-PCR. (H) The expression of miR-31 in CRC cells in parent and 5-FU-resistant CRC cells was examined by qRT-PCR. Data represent the mean $\pm \mathrm{SD}$ from three independent experiments; ${ }^{*} \mathrm{P}<0.05,{ }^{* *} \mathrm{P}<0.01$.

Fig. 3A, ENST00000547547 had a putative binding site for miR-31. Based on this bioinformatic prediction, psiCHECK 2 containing the putative binding sequence and the mutant one were constructed for luciferase assay. The results indicated that miR-31 suppressed the luciferase activity of ENST00000547547-3' in comparison with the negative control, but it had little influence on the mutant both in HCT116 and LoVo cells (Fig. 3B and C). It is suggested that ENST00000547547 interacts with miR-31 via this putative binding site. Recent studies have shown that lncRNAs act as molecular sponges regulating the miRNA activity associatedwith microribonucleoprotein complexes (miRNPs), which play critical roles in cytoplasmic miRNA assembly (28). Ago2 is a core component of the RNA-induced silencing complex (29). For further confirmation, we applied RIP assay to examine the interaction between ENST00000547547 and miR-31 in (Fig. 3D and E). The expression of ENST00000547547 was significantly enriched with anti-AGO2 in comparison with the input and anti-IgG, strongly indicating a direct interaction between ENST00000547547 and miR-31.
Furthermore, we used the ENST00000547547-overexpressed cell lines to investigate whether the expression of miR-31 was regulated by ENST00000547547 (Fig. 2F). We found that the expression of miR-31 was significantly suppressed in the ENST00000547547-overexpressed CRC cell lines LoVo and HCT116 (Fig. 3G). To assess the role of miR-31 in the 5-FU-resistant cell lines, we examined the expression of miR-31 in LoVo/5-FU and HCT116/5-FU cells. As shown in Fig. 3H, the expression of miR-31 was significantly upregulated in LoVo/5-FU and HCT116/5-FU cells compared with their parental cell lines. Collectively, these results indicated that ENST00000547547 could directly bind to miR-31 to suppress its expression, and miR-31 may play an opposing role in 5-FU resistance compared with ENST00000547547.

Knockdown of miR-31 increases the 5-FU sensitivity of CRC cell lines. To explore the role of miR-31 in the 5-FU resistance of CRC cell lines, the miR-31 inhibitor was used for the knockdown of miR-31. As shown in Fig. 4A and B, with various concentrations of 5-FU treatments, both the 
A

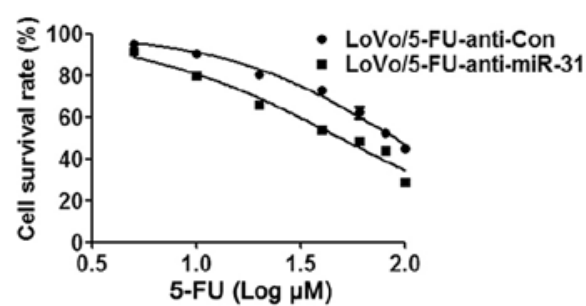

B

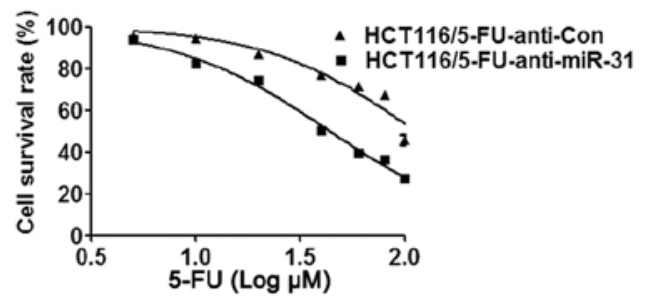

C
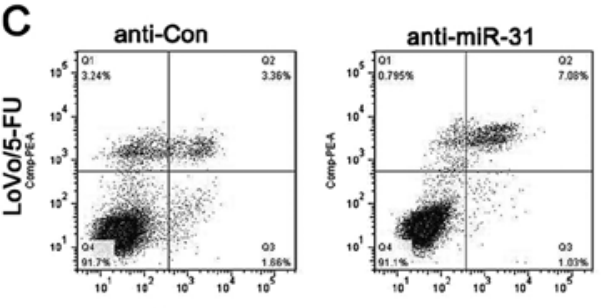

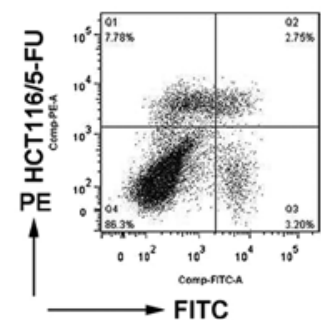

E

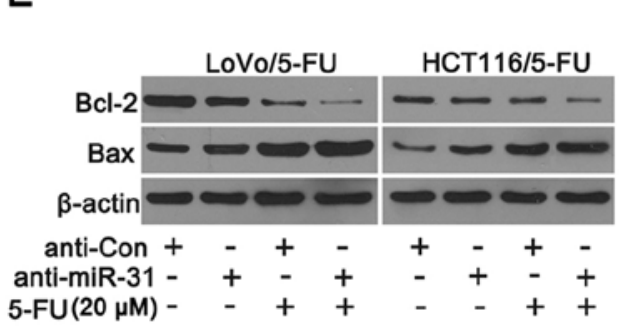

$\mathrm{H}$

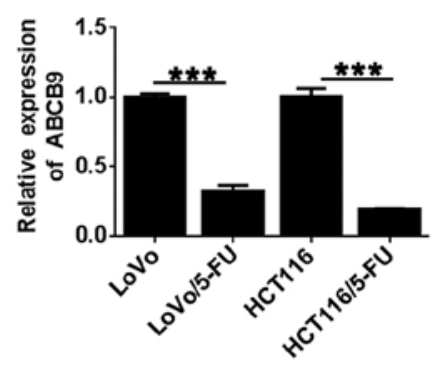

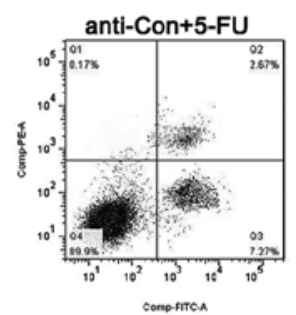
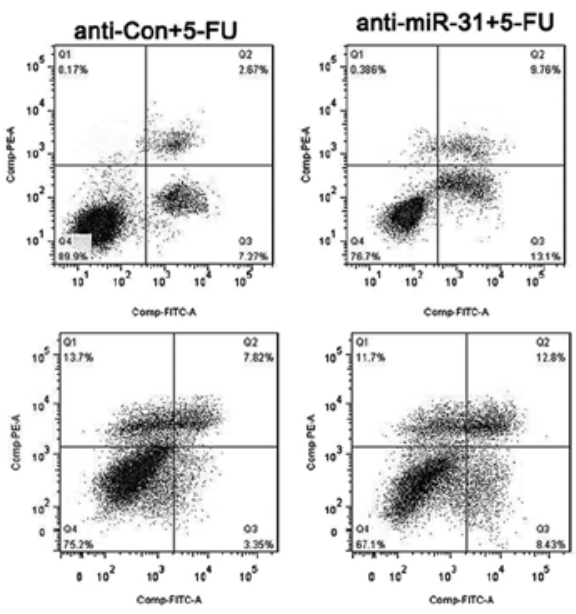

D
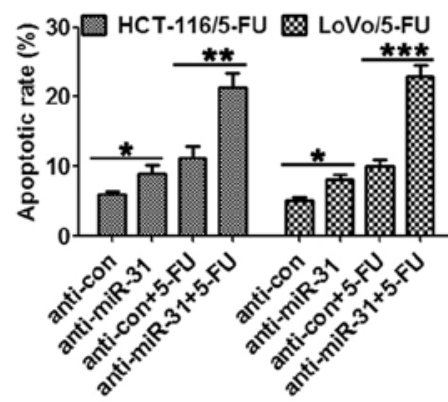

$\square$ LoVo/5-FU $\square$ HCT116/5-FU

$\mathbf{F}$

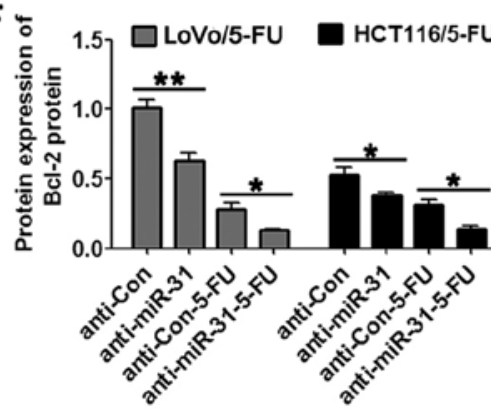

I

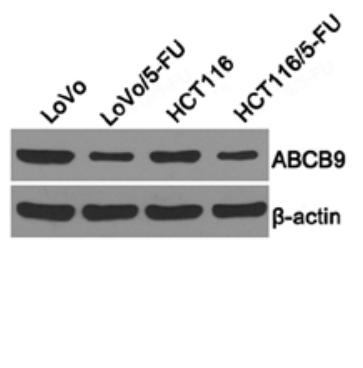

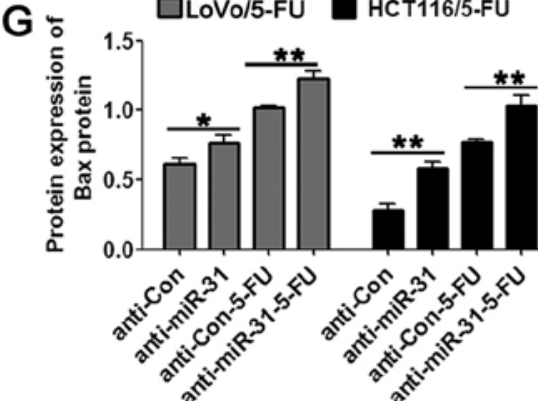

$J$

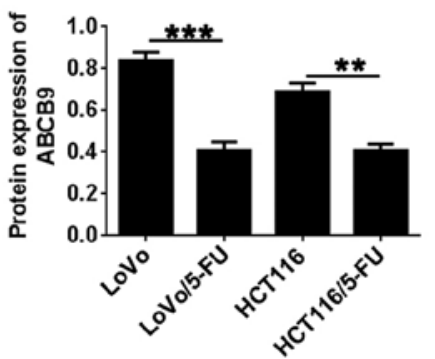

Figure 4. Knockdown of miR-31 increases the 5-FU sensitivity of CRC cell lines. (A and B) The cell survival rate of (A) LoVo/5-FU and (B) HCT116/5-FU cells transfected with a miR-31 inhibitor and a blank inhibitor vector was determined by MTT assay with treatments of 5-FU. (C and D) The apoptotic rates in the miR-31-downregulated CRC cell lines with or without 5-FU treatment were assessed by flow cytometric assays with Annexin V-FITC. (E-G) Western blot analyses were performed to detect the protein expression of Bax and Bcl-2 in the miR-31-downregulated CRC cell lines with or without 5-FU treatment. (H-J) The expression of ABCB9 was examined by (H) qRT-PCR and (I and J) western blot analysis. All experiments were performed in biological triplicates; ${ }^{*} \mathrm{P}<0.05,{ }^{* *} \mathrm{P}<0.01,{ }^{* * *} \mathrm{P}<0.001$.

LoVo/5-FU (Fig. 4A) and HCT116/5-FU (Fig. 4B) transfected with miR-31 inhibitor vectors exhibited lower cell survival rates and reduced 5-FU resistance compared with the negative control. In addition, the $\mathrm{IC}_{50}$ values of $5-\mathrm{FU}$ in the downregulated miR-31 LoVo/5-FU (43.79 $\mu \mathrm{M})$ and HCT116/5-FU $(48.72 \mu \mathrm{M})$ cells were much lower than that of LoVo/5-FU $(112.2 \mu \mathrm{M})$ and HCT116/5-FU $(87.01 \mu \mathrm{M})$ cells. Furthermore, apoptosis assays were performed to investigate whether miR-31 played an antagonistic role in apoptosis compared with ENST00000547547. The results revealed that the apoptotic rates of HCT116/5-FU and LoVo/5-FU cells were significantly improved by knockdown of miR-31 in comparison with the negative control after 5-FU treatment (Fig. 4C and D). Furthermore, using western blot analysis, we found that the expression of anti-apoptotic regulator Bcl-2 and pro-apoptotic regulator Bax were correspondingly affected by knockdown of miR-31 in HCT116/5-FU and LoVo/5-FU cells (Fig. 4E-G). Additionally, since the ATP-binding cassette, sub-family B, member 9 (ABCB9) is a transporter which was reported to be targeted by miR-31, involved in cisplatin-induced apoptosis (26), 
A
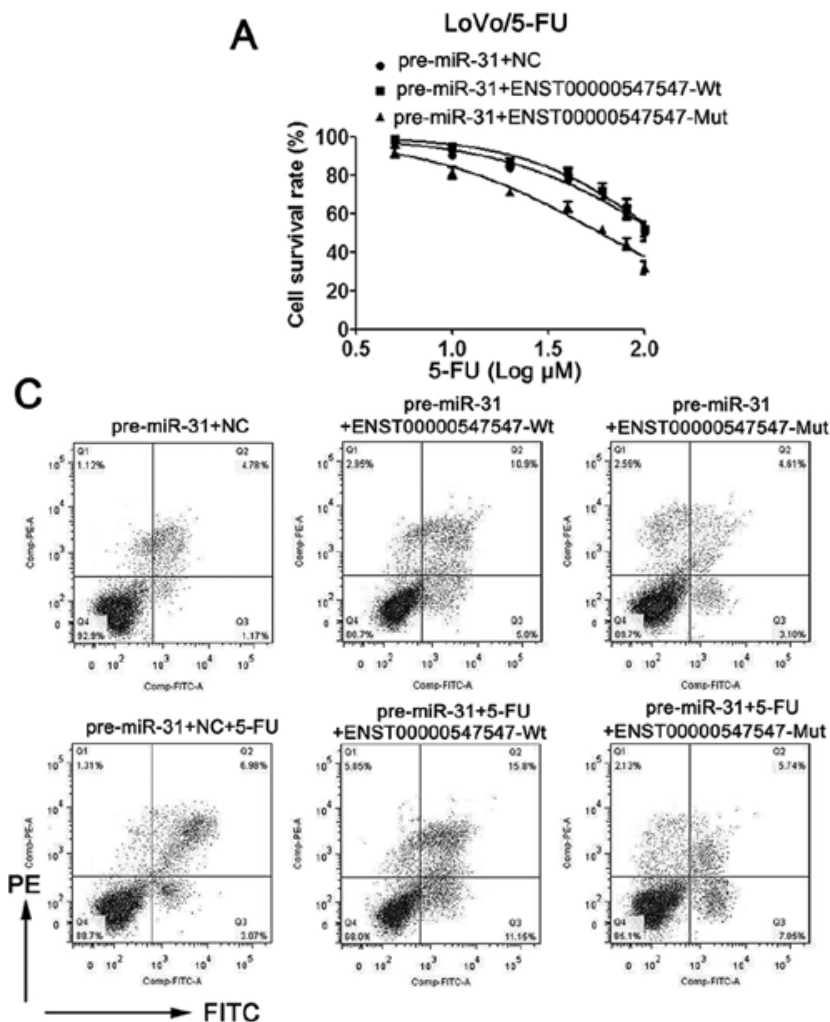

pre-miR-31+5-FU

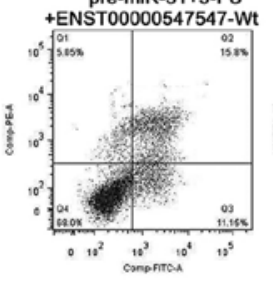

pre-miR-31+5-FU

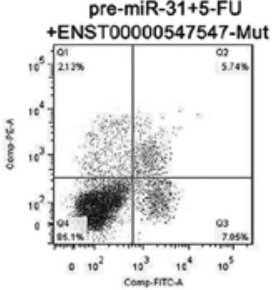

B

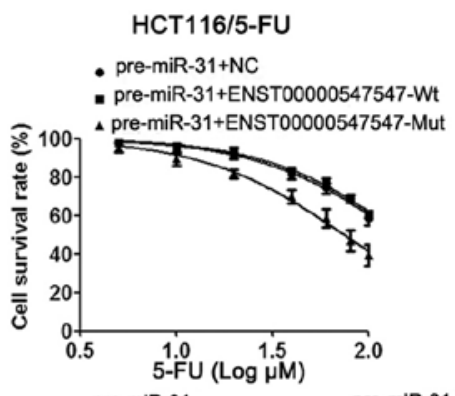

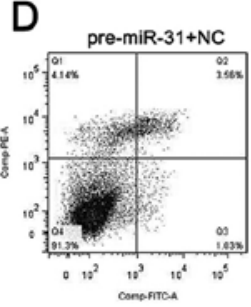

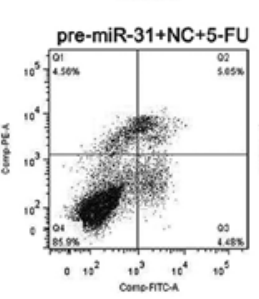

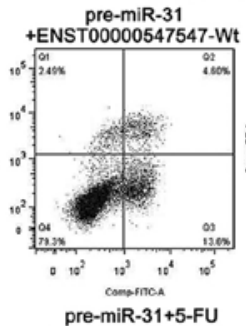

pre-miR-31
+ENST00000547547-Mut

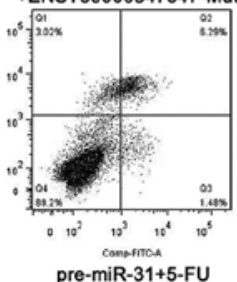

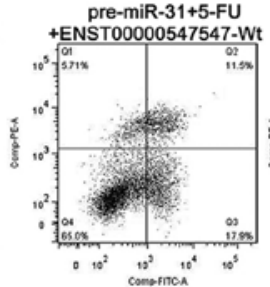

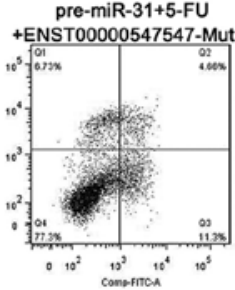

$\mathbf{F}$

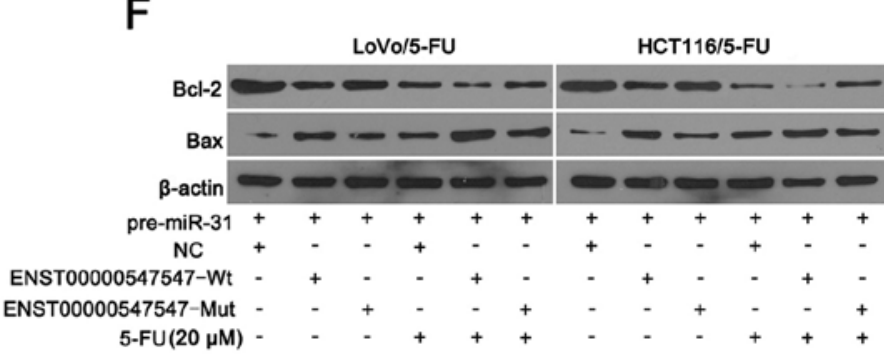

$\mathrm{H}$

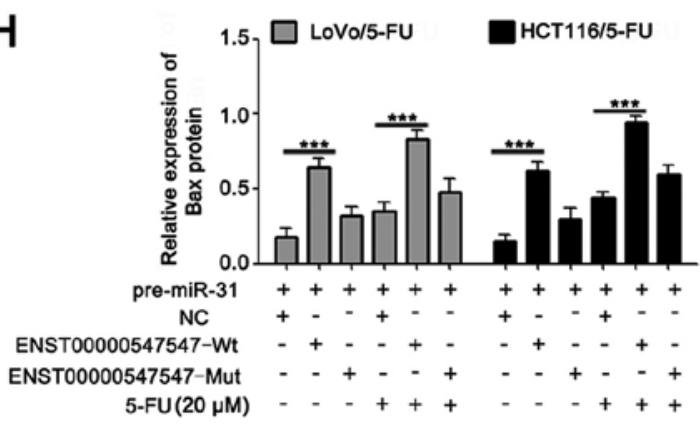

Figure 5. ENST00000547547 reduces the 5-FU resistance via competitive binding to miR-31. (A and B) Cell survival rates were calculated using MTT analysis. (C-E) The apoptosis analysis was conducted in the transfected (C) LoVo/5-FU and (D) HCT116/5-FU cells in miR-31-overexpressing cells in ENST00000547547-Wt or ENST00000547547-Mut with or without exposure to 5-FU for 48 h. (F-H) The protein levels of Bcl-2 and Bax in HCT116/5-FU and $\mathrm{LoVo} / 5$-FU groups were assessed by western blot analysis. The graphs are representative from three independent experiments and display the means $\pm \mathrm{SD}$; ${ }^{*} \mathrm{P}<0.05,{ }^{* *} \mathrm{P}<0.01,{ }^{* * *} \mathrm{P}<0.001$.

we examined the expression of ABCB9 using qRT-PCR and western blot analysis, respectively. The results revealed that ABCB9 was markedly downregulated in HCT116/5-FU and LoVo/5-FU cells (Fig. 4H-J). These data indicated that knockdown of miR-31 increased the 5-FU sensitivity of CRC cells at least partly by upregulation of apoptosis, suggesting that miR-31 plays an antagonistic role in comparison with ENST00000547547 in the regulation of 5-FU resistance.
ENST00000547547 reduces the 5-FU resistance via competitive binding to $\mathrm{miR}$-31. To determine the effect of the interaction between ENST00000547547 and miR-31 in 5-FU resistance, we co-transfected them into HCT116/5-FU and LoVo/5-FU cells. The MTT results indicated that ENST00000547547 overexpression attenuated the positive effect of miR-31 on 5-FU resistance both in LoVo/5-FU (Fig. 5A) and HCT116/5-FU cells (Fig. 5B), whereas the ENST00000547547 


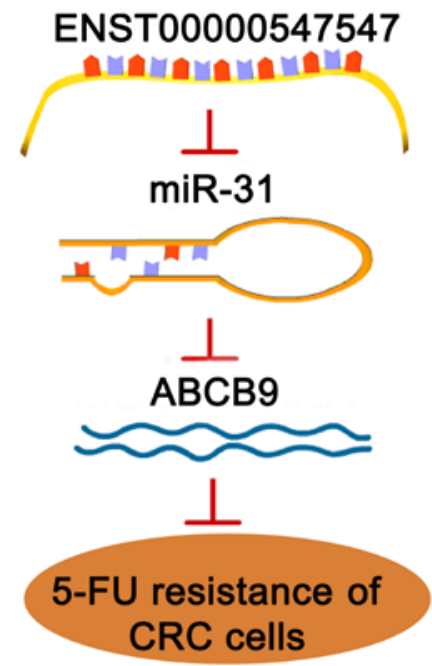

Figure 6. Schematic diagram of mechanism in the present study. ENST00000547547 promotes ABCB9 expression by acting as a sponge of miR-31 and reduces the 5-FU resistance of CRC cells.

with a mutant miR-31 binding site had little influence on the function of miR-31. To further explore whether the interaction between ENST00000547547 and miR-31 affected apoptosis of HCT116/5-FU and LoVo/5-FU cells under 5-FU treatment, we performed flow cytometric assays in these co-transfected CRC cell lines (Fig. 5C-E). The results demonstrated that overexpression of ENST00000547547 suppressed the anti-apoptotic effect of miR-31 via competitive binding to it in LoVo/5-FU and HCT116/5-FU cells. In contrast, the Mut-ENST00000547547 failed to reduce the anti-apoptotic effect of miR-31. Moreover, the expression of anti-apoptotic regulator Bcl-2 was reduced and pro-apoptotic regulator Bax was significantly improved in LoVo/5-FU and HCT116/5-FU cells that were transfected with pre-miR-31 and ENST00000547547 (Fig. 5F-H). Collectively, these results indicated that ENST00000547547 reduces the 5-FU resistance via competitive binding to miR-31 in CRC cells (Fig. 6).

\section{Discussion}

5-Fluorouracil (5-FU) is one of the most widely used anticancer agents for decades now, and 5-FU-based chemotherapy is recommended as the standard treatment for colorectal cancer (CRC) (30). It is well known that 5-FU enhances ROS production and induces marked apoptosis in 5-FU-sensitive cancer cells (31). However, the main obstacle to effective therapy with 5-FU is drug resistance (32). A large number of altered gene expression was found in the 5-FU-resistant CRC cells, and the microarray analyses of IncRNA and microRNA expression profiles indicated that 5-FU-based chemotherapeutic resistance in CRCs was associated with the changes of IncRNA and microRNA expression (33-35). In the present study, we found that the expression of ENST00000547547 was downregulated and miR-31 exhibited significant upregulation in the 5-FU-resistant CRC cells compared to the parent cells. In addition, ENST00000547547 reduced chemoresistance of 5-FU-resistant CRC cells through competitive binding to miR-31.

In the present study, we demonstrated for the first time that ENST00000547547 expression was downregulated in 5-FU-resistant CRC cell lines in comparison with the parental cell lines. In a previous study, the dysregulations of lncRNAs were found to be involved in the regulation of proliferation, apoptosis and drug sensitivity in human cancers (36). Cheng et al reported that overexpression of IncRNA HULC promoted proliferation and invasion, and it inhibited cell apoptosis in gastric cancer cells (9). Whereas, Pickard and Williams found that the expression of 1ncRNA GAS5 was positively correlated to cell responses to apoptotic stimuli, including chemotherapeutic agents, and the expression of GAS5 was directly proportional to the extent of cancer cell death (37). Similar to the functions of GAS5, our results indicated that overexpression of ENST00000547547 promoted apoptotic rates and improved the 5-FU sensitivity in 5-FU-resistant CRC cell lines, suggesting that ENST00000547547 played a positive role in enhancing the 5-FU sensitivity of 5-FU-resistant CRC cells.

The functions of microRNAs in tumor development and drug sensitivity have been extensively studied in recent years. Borralho et al demonstrated that miR-143 was downregulated in colon cancer, and upregulation of miR-143 was associated with increased sensitivity to 5-FU in CRC cells (32). Conversely, Valeri et al found that overexpression of miR-21 markedly reduced sensitivity to 5-FU and undermined the therapeutic efficacy of 5-FU in CRC cells (6). In a previous study, $\mathrm{Xu}$ et al found that expression of miR-31, along with miR-21, miR-96 and miR-135b, was upregulated in the CRC tissues compared to normal mucosal tissues (38). In addition, Wang et al indicated that suppression of miR-31 alone had no effect on cell proliferation. Notably, it improved the 5-FU sensitivity of CRC cell line HCT116 at an early stage (25). In the present study, our results demonstrated that knockdown of miR-31 reduced 5-FU resistance of both 5-FU-resistant CRC cell lines HCT116/5-FU and LoVo/5-FU, and upregulated apoptosis of these CRC cells, suggesting that miR-31 plays an antagonistic role in the regulation of 5-FU sensitivity, compared with ENST00000547547.

The RNA transcripts, including mRNAs, circular RNAs and lncRNAs have been identified as competing endogenous RNAs, which function as molecular sponges to inhibit the endogenous suppressive effect of their interacting microRNAs on target genes $(39,40)$. In hepatocellular carcinoma, Deng et al reported that upregulation of IncRNA CCAT1 was correlated with tumor size, microvascular invasion and poor prognosis, and it promoted hepatocellular carcinoma progression by competitively binding to microRNA let-7 (41). In gastric cancer, Zhou et al revealed that miR-141 formed complementary base pairing with lncRNA H19, and the interaction between them induced translational suppression of a RLuc-H19 reporter gene (42). In CRC, particularly in drug resistance, the importance of the interaction between IncRNAs and microRNAs has rarely been addressed. Our results demonstrated that transcript levels of ENST00000547547 and miR-31 were negatively correlated in CRC cells. In addition, we indicated a direct interaction between ENST00000547547 and miR-31 using dual-luciferase reporter and RIP assays. Moreover, we clarified that by competitive binding to miR-31, ENST00000547547 inhibited the effect of miR-31, which was reducing the 5-FU sensitivity and apoptosis of CRC cells, and ENST00000547547 played a positive role in promoting 5-FU sensitivity of CRC cells. 
Although the present study still has some short comings it may still provide some direction for further research. Firstly, we only demonstrated that one InRNA reduced resistance to one drug. However, in our preliminary experiment, we found that oxaliplatin also upregulated the expression of ENST00000547547, only its effect was lower than that of 5-FU (data not shown). Secondly, we only found that Bax and Bcl-2 played an important role in the regulation of cell apoptosis of 5-FU-resistant CRC cells. Further apoptosis makers and mechanisms need to be examined. In addition, knockdown of miR-31 may upregulate the expression of lncRNA ENST00000547547 due to the competitive relationship of ENST00000547547/ miR-31/ABCB9, however this needs to be ascertained by further experiments (43-45). Furthermore, animal experiments and the mechanism of 5-FU resistance in CRC for patients receiving clinical treatment also need to be researched in the future.

In conclusion, the present study demonstrated for the first time that IncRNA ENST00000547547 expression was downregulated in 5-FU-resistant CRC cells, and its overexpression was associated with the reduction of 5-FU resistance. Knockdown of miR-31 improved 5-FU sensitivity and the apoptotic rate of 5-FU resistance of CRC cells. Furthermore, ENST00000547547 promoted 5-FU sensitivity of 5-FU-resistant CRC cells by directly binding to miR-31. Collectively, our findings indicated that lncRNA ENST00000547547 may be a positive prognostic factor for 5-FU-based chemotherapy and is a potential target for CRC therapy.

\section{Acknowledgements}

The present study was supported by the New Xiangya Talent Project of the Third Xiangya Hospital of Central South University (no. JY201508).

\section{References}

1. Ferlay J, Soerjomataram I, Dikshit R, Eser S, Mathers C, Rebelo M, Parkin DM, Forman D and Bray F: Cancer incidence and mortality worldwide: Sources, methods and major patterns in GLOBOCAN 2012. Int J Cancer 136: E359-E386, 2015.

2. Heidelberger C, Chaudhuri NK, Danneberg P, Mooren D, Griesbach L, Duschinsky R, Schnitzer RJ, Pleven E and Scheiner J: Fluorinated pyrimidines, a new class of tumourinhibitory compounds. Nature 179: 663-666, 1957.

3. Malet-Martino M and Martino R: Clinical studies of three oral prodrugs of 5-fluorouracil (capecitabine, UFT, S-1): A review. Oncologist 7: 288-323, 2002.

4. Sebag-Montefiore D, Glynne-Jones R, Falk S, Meadows HM and Maughan T: A phase I/II study of oxaliplatin when added to 5-fluorouracil and leucovorin and pelvic radiation in locally advanced rectal cancer: A Colorectal Clinical Oncology Group (CCOG) study. Br J Cancer 93: 993-998, 2005.

5. Tsuruta M, Nishibori H, Hasegawa H, Ishii Y, Endo T, Kubota T, Kitajima M and Kitagawa Y: Heat shock protein 27, a nove regulator of 5-fluorouracil resistance in colon cancer. Oncol Rep 20: 1165-1172, 2008.

6. Valeri N, Gasparini P, Braconi C, Paone A, Lovat F, Fabbri M, Sumani KM, Alder H, Amadori D, Patel T, et al: MicroRNA-21 induces resistance to 5-fluorouracil by down-regulating human DNA MutS homolog 2 (hMSH2). Proc Natl Acad Sci USA 107: 21098-21103, 2010.

7. Longley DB, Harkin DP and Johnston PG: 5-Fluorouracil: Mechanisms of action and clinical strategies. Nat Rev Cancer 3: 330-338, 2003.

8. Su X, Malouf GG, Chen Y, Zhang J, Yao H, Valero V, Weinstein JN, Spano JP, Meric-Bernstam F, Khayat D, et al: Comprehensive analysis of long non-coding RNAs in human breast cancer clinical subtypes. Oncotarget 5: 9864-9876, 2014.
9. Cheng N, Li X, Zhao C, Ren S, Chen X, Cai W, Zhao M, Zhang Y, Li J, Wang Q, et al: Microarray expression profile of long noncoding RNAs in EGFR-TKIs resistance of human non-small cell lung cancer. Oncol Rep 33: 833-839, 2015.

10. Luo J, Xu L, Jiang Y, Zhuo D, Zhang S, Wu L, Xu H and Huang Y: Expression profile of long non-coding RNAs in colorectal cancer: A microarray analysis. Oncol Rep 35: 2035-2044, 2016.

11. Tano $\mathrm{K}$ and Akimitsu N: Long non-coding RNAs in cancer progression. Front Genet 3: 219, 2012.

12. Malek E, Jagannathan S and Driscoll JJ: Correlation of long non-coding RNA expression with metastasis, drug resistance and clinical outcome in cancer. Oncotarget 5: 8027-8038, 2014.

13. Wang Q, Cheng N, Li X, Pan H, Li C, Ren S, Su C, Cai W, Zhao C, Zhang L, et al: Correlation of long non-coding RNA $H 19$ expression with cisplatin-resistance and clinical outcome in lung adenocarcinoma. Oncotarget 8: 2558-2567, 2017.

14. Wang Y, Wu K, Yang Z, Zhao Q, Fan D, Xu P, Nie Y and Fan D: Multidrug-resistance related long non-coding RNA expression profile analysis of gastric cancer. PLoS One 10: e0135461, 2015.

15. Pan J, Li X, Wu W, Xue M, Hou H, Zhai W and Chen W Long non-coding RNA UCA1 promotes cisplatin/gemcitabine resistance through CREB modulating miR-196a-5p in bladder cancer cells. Cancer Lett 382: 64-76, 2016.

16. Lan WG, Xu DH, Xu C, Ding CL, Ning FL, Zhou YL, Ma LB, Liu CM and Han X: Silencing of long non-coding RNA ANRIL inhibits the development of multidrug resistance in gastric cancer cells. Oncol Rep 36: 263-270, 2016.

17. Liu E, Liu Z, Zhou Y, Mi R and Wang D: Overexpression of long non-coding RNA PVT1 in ovarian cancer cells promotes cisplatin resistance by regulating apoptotic pathways. Int J Clin Exp Med 8: 20565-20572, 2015.

18. Wang F, Zhou J, Xie X, Hu J, Chen L, Hu Q, Guo H and Yu C: Involvement of SRPK1 in cisplatin resistance related to long non-coding RNA UCA1 in human ovarian cancer cells. Neoplasma 62: 432-438, 2015.

19. Yang L, Qiu M, Xu Y, Wang J, Zheng Y, Li M, Xu L and Yin R: Upregulation of long non-coding RNA PRNCR1 in colorectal cancer promotes cell proliferation and cell cycle progression. Oncol Rep 35: 318-324, 2016

20. Xu C, Yang M, Tian J, Wang X and Li Z: MALAT-1: A long non-coding RNA and its important 3 ' end functional motif in colorectal cancer metastasis. Int J Oncol 39: 169-175, 2011.

21. Kogo R, Shimamura T, Mimori K, Kawahara K, Imoto S, Sudo T, Tanaka F, Shibata K, Suzuki A, Komune S, et al: Long noncoding RNA HOTAIR regulates polycomb-dependent chromatin modification and is associated with poor prognosis in colorectal cancers. Cancer Res 71: 6320-6326, 2011.

22. Niu H, Hu Z, Liu H, Hu G, Yang B, Wu S and Li F: Long non-coding RNA AK027294 involves in the process of proliferation, migration, and apoptosis of colorectal cancer cells. Tumour Biol 37: 10097-10105, 2016.

23. Xiong W, Jiang YX, Ai YQ, Liu S, Wu XR, Cui JG, Qin JY, Liu Y, $\mathrm{Xia} \mathrm{YX}, \mathrm{Ju} \mathrm{YH}$, et al: Microarray analysis of long non-coding RNA expression profile associated with 5-fluorouracil-based chemoradiation resistance in colorectal cancer cells. Asian Pac J Cancer Prev 16: 3395-3402, 2015.

24. Liu X, Cheng Y, Chen X, Yang J, Xu L and Zhang C: MicroRNA-31 regulated by the extracellular regulated kinase is involved in vascular smooth muscle cell growth via large tumor suppressor homolog 2. J Biol Chem 286: 42371-42380, 2011.

25. Wang CJ, Stratmann J, Zhou ZG and Sun XF: Suppression of microRNA-31 increases sensitivity to 5-FU at an early stage, and affects cell migration and invasion in HCT-116 colon cancer cells. BMC Cancer 10: 616, 2010.

26. Dong Z, Zhong Z, Yang L, Wang S and Gong Z: MicroRNA-31 inhibits cisplatin-induced apoptosis in non-small cell lung cancer cells by regulating the drug transporter ABCB9. Cancer Lett 343: 249-257, 2014

27. Miyashita T, Krajewski S, Krajewska M, Wang HG, Lin HK, Liebermann DA, Hoffman B and Reed JC: Tumor suppressor p53 is a regulator of bcl-2 and bax gene expression in vitro and in vivo. Oncogene 9: 1799-1805, 1994.

28. Gong C, Popp MW and Maquat LE: Biochemical analysis of long non-coding RNA-containing ribonucleoprotein complexes. Methods 58: 88-93, 2012.

29. Hou P, Zhao Y, Li Z, Yao R, Ma M, Gao Y, Zhao L, Zhang Y, Huang B and Lu J: LincRNA-ROR induces epithelial-to-mesenchymal transition and contributes to breast cancer tumorigenesis and metastasis. Cell Death Dis 5: e1287, 2014. 
30. Hwang IT, Chung YM, Kim JJ, Chung JS, Kim BS, Kim HJ, Kim JS and Yoo YD: Drug resistance to 5-FU linked to reactive oxygen species modulator 1. Biochem Biophys Res Commun 359: 304-310, 2007.

31. Hwang PM, Bunz F, Yu J, Rago C, Chan TA, Murphy MP, Kelso GF, Smith RA, Kinzler KW and Vogelstein B: Ferredoxin reductase affects p53-dependent, 5-fluorouracil-induced apoptosis in colorectal cancer cells. Nat Med 7: 1111-1117, 2001

32. Borralho PM, Kren BT, Castro RE, da Silva IB, Steer CJ and Rodrigues CM: MicroRNA-143 reduces viability and increases sensitivity to 5-fluorouracil in HCT116 human colorectal cancer cells. FEBS J 276: 6689-6700, 2009.

33. Choi S and Ku JL: Resistance of colorectal cancer cells to radiation and 5-FU is associated with MELK expression. Biochem Biophys Res Commun 412: 207-213, 2011.

34. Spitzner M, Emons G, Kramer F, Gaedcke J, Rave-Fränk M, Scharf JG, Burfeind P, Becker H, Beissbarth T, Ghadimi BM, et al: A gene expression signature for chemoradiosensitivity of colorectal cancer cells. Int J Radiat Oncol Biol Phys 78: 1184-1192, 2010.

35. Fu J, Tang W, Du P, Wang G, Chen W, Li J, Zhu Y, Gao J and Cui L: Identifying microRNA-mRNA regulatory network in colorectal cancer by a combination of expression profile and bioinformatics analysis. BMC Syst Biol 6: 68, 2012.

36. Diederichs S: Long non-coding RNA \& cancer. In: Proceedings of the 37th World Congress of the International Union of Physiological Sciences. Birmingham, UK, 2013.

37. Pickard MR and Williams GT: Regulation of apoptosis by long non-coding RNA GAS5 in breast cancer cells: Implications for chemotherapy. Breast Cancer Res Treat 145: 359-370, 2014.

38. Xu XM, Qian JC, Deng ZL, Cai Z, Tang T, Wang P, Zhang KH and Cai JP: Expression of miR-21, miR-31, miR-96 and miR-135b is correlated with the clinical parameters of colorectal cancer. Oncol Lett 4: 339-345, 2012.
39. Cesana M, Cacchiarelli D, Legnini I, Santini T, Sthandier O, Chinappi M, Tramontano A and Bozzoni I: A long noncoding RNA controls muscle differentiation by functioning as a competing endogenous RNA. Cell 147: 358-369, 2011.

40. Memczak S, Jens M, Elefsinioti A, Torti F, Krueger J, Rybak A, Maier L, Mackowiak SD, Gregersen LH, Munschauer M, et al: Circular RNAs are a large class of animal RNAs with regulatory potency. Nature 495: 333-338, 2013.

41. Deng L, Yang SB, Xu FF and Zhang JH: Long noncoding RNA CCAT1 promotes hepatocellular carcinoma progression by functioning as let-7 sponge. J Exp Clin Cancer Res 34: 18, 2015.

42. Zhou X, Ye F, Yin C, Zhuang Y, Yue G and Zhang G: The Interaction between miR-141 and lncRNA-H19 in regulating cell proliferation and migration in gastric cancer. Cell Physiol Biochem 36: 1440-1452, 2015.

43. Cai H, Yao J, An Y, Chen X, Chen W, Wu D, Luo B, Yang Y, Jiang Y, Sun D, et al: LncRNA HOTAIR acts a competing endogenous RNA to control the expression of notch3 via sponging miR-613 in pancreatic cancer. Oncotarget 8: 32905-32917, 2017.

44. Yu F, Lu Z, Cai J, Huang K, Chen B, Li G, Dong P and Zheng J: MALAT1 functions as a competing endogenous RNA to mediate Racl expression by sequestering miR-101b in liver fibrosis. Cell Cycle 14: 3885-3896, 2015.

45. Wang SH, Zhang WJ, Wu XC, Weng MZ, Zhang MD, Cai Q, Zhou D, Wang JD and Quan ZW: The IncRNA MALAT1 functions as a competing endogenous RNA to regulate MCL-1 expression by sponging miR-363-3p in gallbladder cancer. J Cell Mol Med 20: 2299-2308, 2016. 